\title{
The Global Distribution of Nitric Oxide in the Thermosphere as Determined by the Atmosphere Explorer D Satellite
}

\author{
T. E. Cravens \\ Space Physics Research Laboratory, Atmospheric and Oceanic Sciences Department, The University of Michigan, Ann Arbor \\ J.-C. GÉRARD \\ Institut d'Astrophysique, Université de Liège, Cointe-Liège, Belgium \\ M. LeCompte, A. I. STeWART, AND D. W. Rusch \\ Laboratory for Atmospheric and Space Physics, Department of Astrophysical, Planetary and Atmospheric Sciences \\ University of Colorado, Boulder

\begin{abstract}
The ultraviolet nitric oxide spectrometer (UVNO) experiment on the Atmosphere Explorer D (AE-D) satellite measured thermospheric nitric oxide during the winter of 1974-1975 using resonant fluorescence from the 1-0 gamma band of the molecule. Almost complete latitude coverage was obtained, but the observations were confined to morning local times close to 0900 . The 1-0 gamma band intensity profiles measured by the instrument were inverted to provide vertical profiles of the NO number density between about 90 and $200 \mathrm{~km}$. Typically, the measured NO concentrations reached a maximum between altitudes of 100 and $110 \mathrm{~km}$, and more NO was observed at higher latitudes than at low latitudes, in agreement with previous observational studies. The shape of the NO profile was also found to be a function of latitude, with a plateau appearing in the profile near $130 \mathrm{~km}$ for low latitudes and mid-latitudes in the winter hemisphere.
\end{abstract}

\section{INTRODUCTION}

Nitric oxide density profiles for thermospheric altitudes have been measured by experiments on many rocket flights over the last decade or two [c.f. Gérard et al., 1983; McCoy, 1983a]. These rocket experiments have provided detailed information on the altitude distribution of NO at particular times and locations. However, NO is quite variable both spatially and temporally, which makes global observations of it desirable.

OGO 4 measured NO both at mid-latitudes and in the auroral region [Rusch, 1973; Rusch and Barth, 1975; Gérard and Barth, 1977]. Nitric oxide tangential column densities for thermospheric altitudes were also measured by the ultraviolet spectrometers (UVNO) on both the Atmosphere Explorer $\mathbf{C}$ and $D$ satellites (AE-C and AE-D). These instruments, which were described by Barth et al. [1973], measured sunlight fluorescently scattered in the 1-0 gamma band of the molecule at 2150 A. Stewart and Cravens [1978] analyzed NO data from AE-C for $105 \mathrm{~km}$ for magnetically quiet times, and Cravens and Stewart [1978] did the same for higher levels of magnetic activity. Cravens et al. [1979] further studied NO at $105 \mathrm{~km}$ but also presented data for NO at $200 \mathrm{~km}$ from orbits during which AE-C was despun. Cravens [1981] presented virtually all despun AE-D data for an altitude of $200 \mathrm{~km}$. All these satellite studies emphasized the horizontal and temporal variability of nitric oxide, whereas the rocket studies emphasized the details of the vertical distribution.

Both rocket and satellite studies have demonstrated that NO is almost always more abundant at higher latitudes, particularly in the auroral regions where large concentrations of

Copyright 1985 by the American Geophysical Union.

Paper number 4A8414.

0148-0227/85/004A-8414\$05.00
NO in excess of $10^{8} \mathrm{~cm}^{-3}$ have been observed. Cravens and Stewart [1978] determined the manner in which NO in the lower thermosphere (i.e., at $105 \mathrm{~km}$, which is near the peak of a typical NO altitude distribution) varies with latitude, longitude, and magnetic activity. Stewart and Cravens [1978] explored the local time and seasonal variations of NO. These studies showed that NO at middle and high, and sometimes even low, latitudes is correlated with the $A p$ index of magnetic activity. A large latitudinal gradient of NO is present during magnetically active times.

Cravens et al. [1979] demonstrated that these large observed latitudinal gradients could not be explained using the current one-dimensional theories of thermospheric odd nitrogen, and they concluded that significant horizontal transport from the auroral regions to lower latitudes is required. Recently, horizontal as well as vertical transport of odd nitrogen species has been incorporated into some twodimensional theoretical models [Roble and Gary, 1979; Gérard et al., 1984]. The Gérard et al. [1984] calculations are now able to explain more of the observed features of thermospheric NO, such as the general nature of the latitudinal gradients.

Even the current generation of theoretical models does not reproduce all the features of the observed behavior of NO, including the detailed shape of the altitude profiles in some cases. For example, there are no published theoretical vertical profiles [e.g., Oran et al., 1975; Rusch et al., 1977; Cravens et al., 1979; Rusch and Sharp, 1981; McCoy, 1983b; Gérard et al., 1984] which closely resemble the NO profile measured by Thomas [1978] or many of those which will be presented in this paper, although the two-dimensional model of Gérard et al. [1984] is partially successful. There have been no systematic observational studies of how the shape of the NO altitude profile, and not just the concentration at particular altitudes, depends on parameters such as latitude, local time, longitude, and magnetic activity level.

In this paper we present a systematic study of NO profiles 
from about $90 \mathrm{~km}$ to $200 \mathrm{~km}$ for a range of latitudes and magnetic activity levels. We use measurements of nitric oxide from orbits of AE-D for which the satellite was spinning and was thus capable of making altitude scans below the spacecraft altitude.

\section{Measurements and Data Analysis}

The UVNO instrument on AE-D made measurements during a period of time from late November 1975, when the location of the AE-D perigee emerged from darkness near $70^{\circ} \mathrm{N}$ latitude, until February 2, 1976, when the perigee reached the south pole. This time period was during solar cycle minimum for solar cycle 20 and had an average $F 10.7$ flux of 73. The AE-D satellite was in a near-polar orbit, and consequently, very good latitude coverage was obtained. The satellite was spinning during approximately half of the orbits in this time period. The local time changed from about 1000 near $70^{\circ} \mathrm{N}$ to about 0530 at the south pole. All the data considered in this paper can be characterized by morning solstice conditions.

The AE-D satellite spin rate was 4 revolutions per minute around an axis perpendicular to the orbital plane. The limb was scanned twice per spin period by the UVNO instrument so that two profiles of the NO 1-0 gamma band intensity versus tangent ray height were obtained for each spin cycle. The signal for tangent ray altitudes below about $90 \mathrm{~km}$ was contaminated to some degree by Rayleigh scattered light, although some data sets were corrected for this and thus provide useful gamma band intensities down to $75 \mathrm{~km}$.

The signal integration time of the instrument was $21 \mathrm{~ms}$, and the field of view was $0.25^{\circ}$. These parameters limited the vertical resolution obtainable by the instrument when observing an atmospheric layer with a tangent ray height (trh) of 105 $\mathrm{km}$ to approximately $10 \mathrm{~km}$ for spacecraft altitudes close to $200 \mathrm{~km}$ and to approximately $7 \mathrm{~km}$ for a spacecraft altitude of $150 \mathrm{~km}$. Consequently, for this paper we did not use data collected when the satellite was above $200 \mathrm{~km}$ (or $210 \mathrm{~km}$ for some data sets).

Cravens [1977] derived a relation between the slant column density of nitric oxide and the intensity of scattered sunlight from the 1-0 gamma band of the molecule. This relation was convolved with the instrumental sensitivity function in order to give a curve of growth function between the instrumental counts recorded during an integration period and the slant column density of NO. Stewart and Cravens [1978] presented this function for the UVNO spectrometer on the AE-C satellite. The function for the AE-D instrument is virtually identical to the AE-C one if the AE-C function is multiplied by a factor of 1.83. The number of instrumental counts per kiloRayleigh is $\mathbf{4 0 . 3 5}$ for AE-D measurements of optically thin 1-0 gamma band emission.

We have organized the UVNO data from AE-D in several ways. The total number of spin cycles available was close to $10^{4}$, although only a fraction of this total quantity of data was included in any particular data set. The most basic form of the data, which will be discussed in this paper, consists of the instrumental counts (denoted $C$ ) at a particular tangent ray height. Typically, during half of a spin cycle, approximately 25 data points were collected for tangent heights varying from 75 $\mathrm{km}$ up to the spacecraft altitude. These data points were then averaged, filtered, rejected, etc., depending on which type of data set they are being assigned to. The instrumental counts were usually converted into slant column densities of NO using the curve of growth discussed earlier.

Some of the vertical profiles of column density were further inverted to give vertical profiles of NO number density using an inversion routine. The column densities were taken every 5 $\mathrm{km}$ and were then inverted to give NO number densities every $5 \mathrm{~km}$. The effective altitude resolution of the measurements is somewhat greater than $5 \mathrm{~km}$, as discussed above, so that it does not make sense to invert the data at a higher resolution. An Abel-type inversion was utilized with the assumption of spherically symmetric layers of NO. The NO number density was assumed to vary linearly between the discrete altitude values (every $5 \mathrm{~km}$ ). The inversion procedure was checked by integrating the derived number densities along horizontal optical paths. The column densities obtained in this way from the inversion routine agreed almost identically with the original observed values. It was necessary to extrapolate the column density profile (actually the $C$ profiles were extrapolated) to altitudes above the spacecraft altitude for which no data exist. This extrapolation was one of the major sources of error in the inverted profiles and will be discussed more later.

Now we will characterize the different data sets which will be discussed in this paper.

\subsection{Individual NO Profiles}

Each of these data sets consists of data from three consecutive spins of AE-D and will be displayed in the form of both instrumental counts (c) versus tangent ray altitude and NO number density versus altitude. Only data above $90 \mathrm{~km}$ were inverted in order to avoid serious Rayleigh scattering contamination problems.

\subsection{Average NO Column Density Profiles (Contour Plot)}

Data points from a large number of spins were first converted into slant column densities and then sorted into $1^{\circ} \times 1 \mathrm{~km}$ latitude/altitude bins. The data in each bin were then averaged, and a contour map was constructed. Tangent ray altitudes as low as $75 \mathrm{~km}$ were included, but the NO profiles were corrected for Rayleigh scattering contamination. This contribution was subtracted individually from each profile (group of three consecutive spins) by fitting a decreasing exponential curve to the total signal measured near $75 \mathrm{~km}$. The scale height of the exponential is determined from the best fit to five data points in the vicinity of $75 \mathrm{~km}$. The NO gamma band resonance scattering intensity was obtained by subtracting the Rayleigh scattering fitted curve from each of the experimental data points of the observed profile. The maximum permissible satellite altitude was $200 \mathrm{~km}$, and both the ram and the wake parts of spin cycles were included, although only data for which the entire optical ray path was illuminated by the sun were considered acceptable. Finally, a data set containing data points from 3975 satellite spins was compiled.

\subsection{Simplified Inversion}

Data from three consecutive spins, basically the same as those mentioned for the first type of data set, were converted into slant column densities and were then fitted with either a simple three-parameter function or a two-parameter function. These functions are analytically integrable and thus provide a very efficient means of inverting large numbers of column density profiles into number density profiles. The three-parameter function for the number density versus altitude is

$$
n=n_{m}\left\{\exp \left[-\left(z-z_{m}\right) / H_{t}\right]-0.5 \exp \left[-\left(z-z_{m}\right) / 0.5 H_{t}\right]\right\}
$$

$$
n=0 \quad z \leq z_{0}
$$


where

$$
z_{0}=z_{m}-H_{t} \ln (2)
$$

The fitting parameters are the maximum NO concentration $\left(n_{m}\right)$, the altitude of this maximum concentration $\left(z_{m}\right)$, and a single topside scale height $H_{t} . z_{m}$ is set equal to $105 \mathrm{~km}$ and the second term is dropped from equation (1) for the two parameter fit. The 3 parameter function was used only if the fit was better than with the 2 parameter function.

\subsection{Average NO Number Density Profiles (Contour Plot)}

The procedure here was similar to that described for the data set in section 2.2. Data of the type discussed in section 2.1 were averaged using latitude/altitude bins which were $5^{\circ} \times 5$ $\mathrm{km}$, rather than $1^{\circ} \times 1 \mathrm{~km}$ as in section 2.2 . However, only every other latitude bin was used; that is, averaged data are available every $10^{\circ}$ in latitude. This data set contained almost 2000 spins. Average values of the instrumental counts were thus obtained as a function of altitude and latitude. Next, these profiles were converted into column densities. The average column density profiles, which are specified at 5-km intervals, were then inverted at each latitude to give NO number densities. The inversion was applied only above 90 $\mathbf{k m}$ in order to avoid problems with Rayleigh scattering. A contour map of NO number density for altitude and latitude was then constructed from these inverted data.

We have just described the four different ways in which the data have been organized. Now these four different types of data sets will be used to investigate several aspects of the global variation of NO.

\section{Observational Results}

\subsection{Individual NO Profiles}

We will present nine individual NO profiles in this section for several different latitudes and levels of magnetic activity (Figures 1-9). Zonally and temporally averaged NO profiles are displayed later in the paper in a contour plot (Figure 14), which provides a good overview of the average geographical distribution of NO without the effects of time variability, which makes the interpretation of the individual profiles more difficult. Each of the profiles in Figures 1-9 consists of data from three consecutive spins and contains approximately 70 data points. The effective latitudinal extent covered by the three spins due to the orbital motion of AE-D is about $3^{\circ}$. The latitudinal extent of the slant optical path is several degrees. The effective angular (or latitudinal) resolution for a given measurement can be defined as twice the distance between the minimum ray point (or tangent ray point) and the point where the path is one NO scale height, $H$, above the minimum ray height. Using this definition, the effective latitudinal resolution is $6.4^{\circ}$ for $H=10 \mathrm{~km}$ and $9^{\circ}$ for $H=20 \mathrm{~km}$. Hence the effective latitude resolution is approximately $5-10^{\circ}$. In order to obtain good altitude resolution (less than $1 \mathrm{~km}$ for a tangent ray height of $140 \mathrm{~km}$ and about $7 \mathrm{~km}$ for a trh of $100 \mathrm{~km}$ ), data for the profiles shown in figures $1-9$ were taken when the spacecraft was about $152 \mathrm{~km}$ or lower.

Each figure (Figures 1-9) contains a set of data points with a curve smoothly hand-drawn through them. These curves were all extrapolated to altitudes greater than the highest data point, as required by the inversion routine. These extrapolations were made such that the instrumental counts at $200 \mathrm{~km}$ were between 10 and 100 and the scale height was about 30

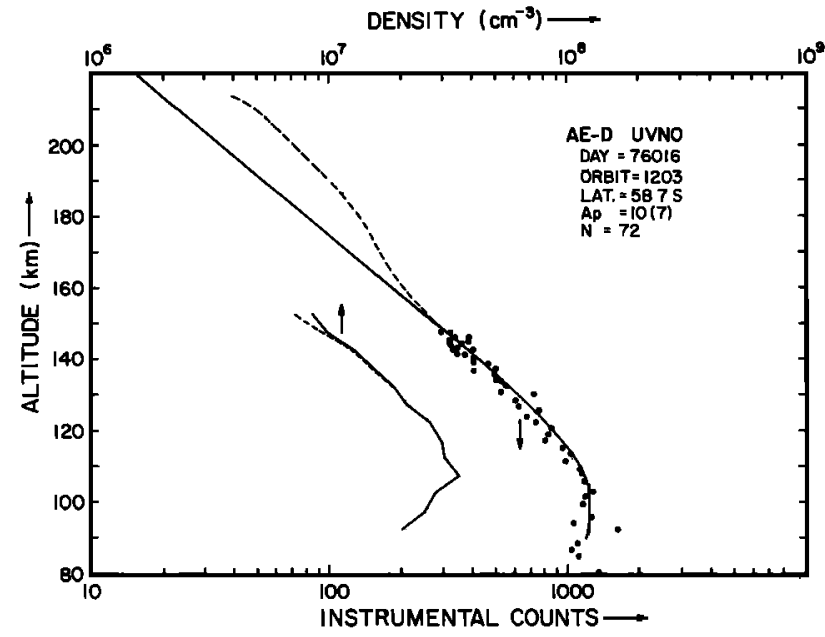

Fig. 1. NO data from three consecutive spins are shown versus minimum ray height. The total number of data points, $N$, is indicated. The data are in the form of counts per instrumental integration period (bottom scale). The statistical uncertainty associated with the instrumental counts themselves is apparently equal to the square root of the counts, and this uncertainty is almost always much smaller than the scatter between data points. The day, latitude of the $105-\mathrm{km}$ minimum ray height, the orbit number, and the $A p$ values for that day and the previous day are indicated. A smooth fit to the data points is also shown (solid line) and includes a reasonable extrapolation to altitudes above the measured points. The dashed line is an alternative high-altitude extrapolation (see text). The lower curves without data points are the result of a numerical inversion of the smooth curves through the data points (top scale). The inversion gives NO number density as a function of altitude.

$\mathrm{km}$, in general agreement with NO measurements actually made at this altitude. Column density values were taken from the curves at $5-\mathrm{km}$ intervals and were then inverted in the manner described in the previous section. The number density profiles derived from the inversion of the instrumental counts profile is also displayed in each figure.

There is very little random error associated with the instrumental counts themselves, but the effective resolution in latitude (about $10^{\circ}$ ) and altitude $(<7 \mathrm{~km}$ ) introduces some scatter in the data points. The statistical uncertainty in the instrumental counts is approximately equal to the square root of the number of instrumental counts, and this is almost always less than $10 \%$ for the data in Figures 1-9. The scatter between data points is indicative of the variability of $\mathrm{NO}$ in time and latitude together with the limited altitude and latitude resolution of the measurements. The major source of error in the inversion procedure is the high-altitude extrapolation. Alternate extrapolations were tried and are shown in some of the figures, and it is apparent that the extrapolations only affect the derived profiles for a few kilometers below the uppermost altitude. The uncertainty in the inverted profile is evident to some extent as sharp irregularities in the profile and is about $20 \%$ near the peak of the NO distribution.

Data from middle to high latitudes in the southern (summer) hemisphere, and for a range of magnetic conditions, are shown in Figures 1-4. The $A p$ index of magnetic activity is indicated on each figure, as well as the index for the previous day because NO in the lower thermosphere usually has a long chemical lifetime [Oran et al., 1975] and responds to changing conditions with a lag time of about a day. The number of data points included in each figure $(N)$ is also indicated.

We will discuss Figure 1 in some detail. The latitude $\left(58.7^{\circ} \mathrm{S}\right)$ is rather high in the summer hemisphere, and the magnetic conditions are quiet. The counts profile (lower scale) 


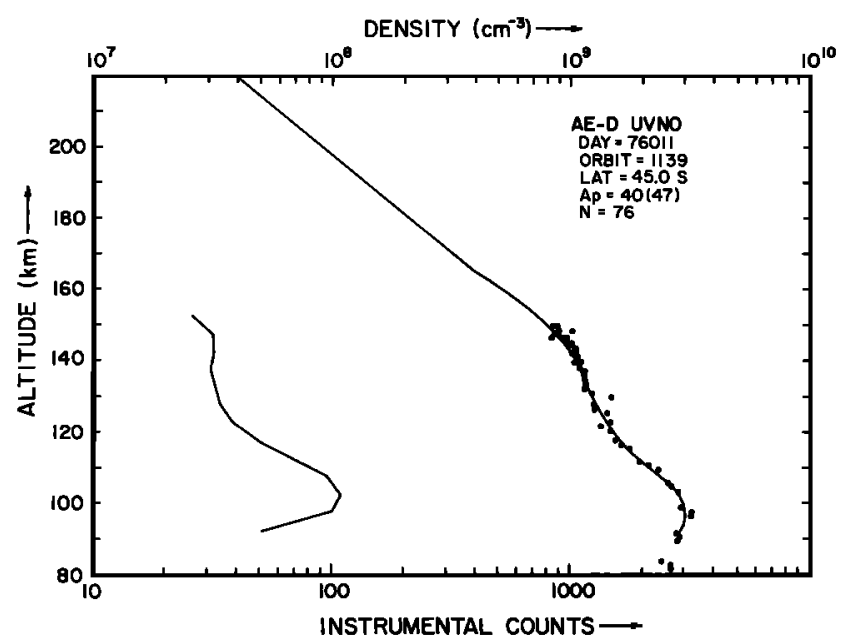

Fig. 2. See Figure 1 caption.

has a broad ledge of 1200 or so below $110 \mathrm{~km}$ and decreases above that altitude with a scale height of about $30 \mathrm{~km} .1200$ AE-D counts are equivalent to $650 \mathrm{AE}-\mathrm{C}$ counts, which was a typical AE-C value at this latitude for quiet conditions [Cravens and Stewart, 1978].

- The number density profile (upper scale) derived from the $C$ profile also has a $30-\mathrm{km}$ scale height at higher altitudes and has a peak density of $3 \times 10^{7} \mathrm{~cm}^{-3}$ at an altitude of $110 \mathrm{~km}$. It is clear from this figure that the number density profile is not very sensitive to the actual choice of the high-altitude extrapolation.

Figure 2 shows a summer mid-latitude profile $\left(45^{\circ} \mathrm{S}\right)$ for magnetically active conditions. $C$ is 3000 at $100 \mathrm{~km}$ and 2500 at $105 \mathrm{~km}$, corresponding to $1350 \mathrm{AE}-\mathrm{C}$ counts at $105 \mathrm{~km}$, which again agrees with the AE-C measurements for this level of activity [Cravens and Stewart, 1978]. The peak concentration is now $10^{8} \mathrm{~cm}^{-3}$ at an altitude of $100-105 \mathrm{~km}$. The density rapidly decreases above the peak and then levels off near $120 \mathrm{~km}$.

Figures 3 and 4 display mid-latitude profiles for quiet conditions. The instrumental counts at $105 \mathrm{~km}$ are quite low, and the peak number densities are between 1 and $3 \times 10^{7} \mathrm{~cm}^{-3}$ near $105 \mathrm{~km}$. Some Rayleigh scattering contribution to $C$ is apparent below $90 \mathrm{~km}$.

Data for low latitudes are shown in Figures 5-7. The peak

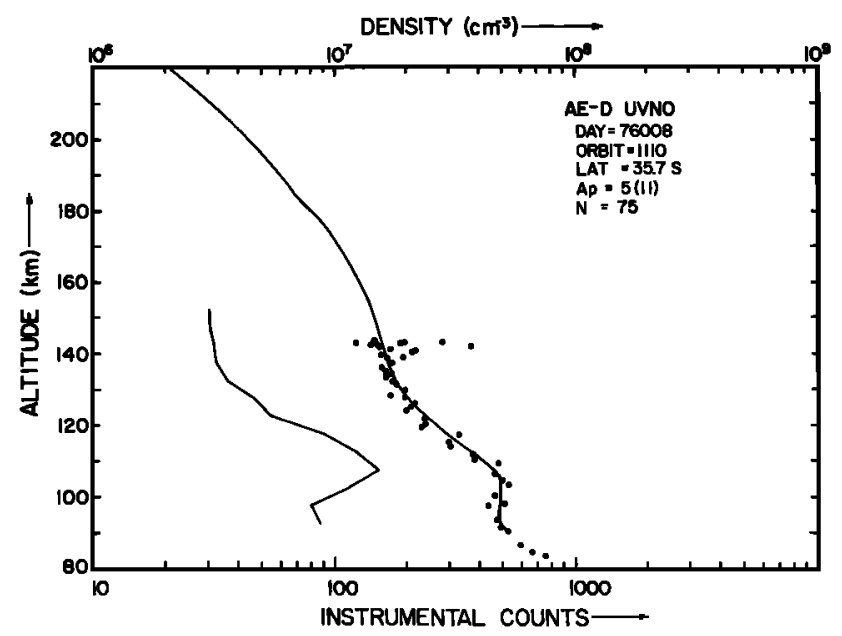

Fig. 4. See Figure 1 caption.

number densities are about $2 \times 10^{7} \mathrm{~cm}^{-3}$ for larger values of $A p$ and about $1 \times 10^{7}$ for quiet conditions. There is again an indication of a leveling off in the densities near $120 \mathrm{~km}$, although for Figure 6 the change in the scale height at this altitude is rather small.

Middle to high northerly (winter) latitudes are represented by Figures 8 and 9. $C$ at $105 \mathrm{~km}$ is $1200-1400$, and the peak density is about $5 \times 10^{7} \mathrm{~cm}^{-3}$ for both sets of data. However, the shapes of the two profiles are quite different. For the low magnetic activity one (Figure 8) the scale height above the peak is very small, with some indication of a ledge at higher altitudes. The peak in Figure 9 (moderate level of activity) is very broad and is located above $120 \mathrm{~km}$. One must interpret high-latitude inverted profiles (like the one shown in Figure 9) with some amount of caution. The inversion method assumes spherical symmetry, and the data themselves have a horizontal resolution of about $10^{\circ}$, but $\mathrm{NO}$ in the auroral regions is known to be quite variable and is expected to have considerable horizontal structure [Rusch and Barth, 1975; Gérard et al., 1983]. The gross features of both the instrumental counts and the number density profiles are certainly correct, but the finer quantitative details of the inverted data are not certain, especially below the maximum where the inversion is more sensitive to relatively small variations in the counts profile than at higher altitudes.

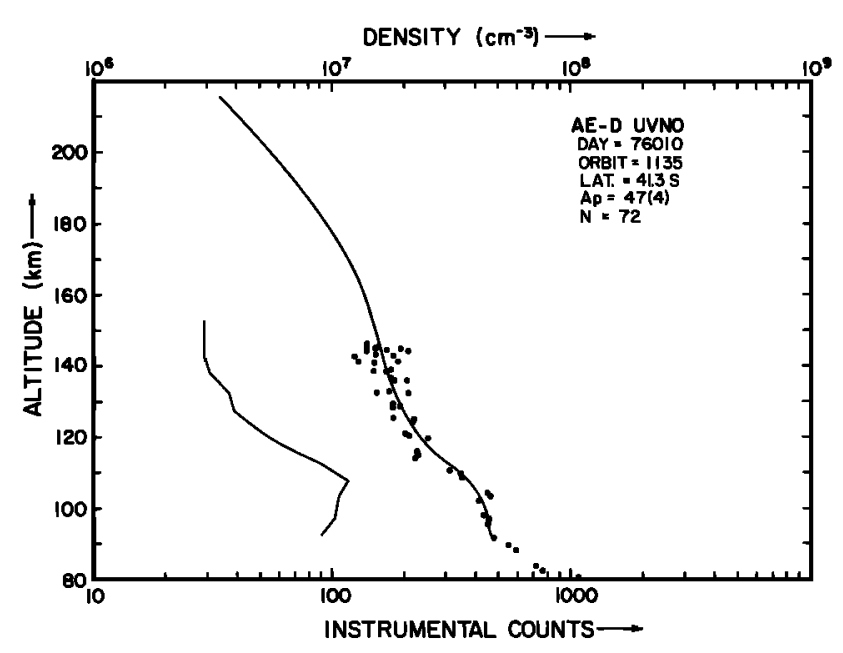

Fig. 3. See Figure 1 caption.

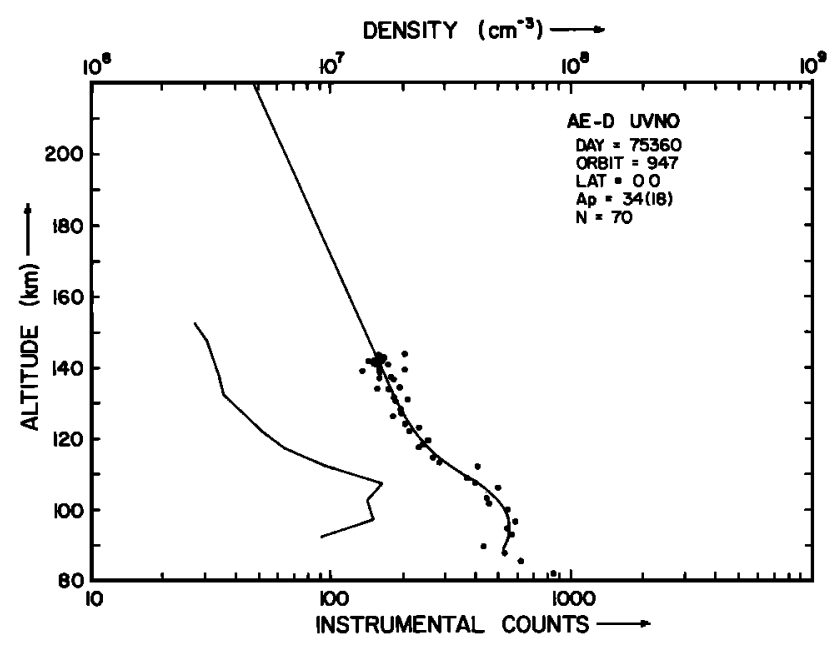

Fig. 5. See Figure 1 caption. 


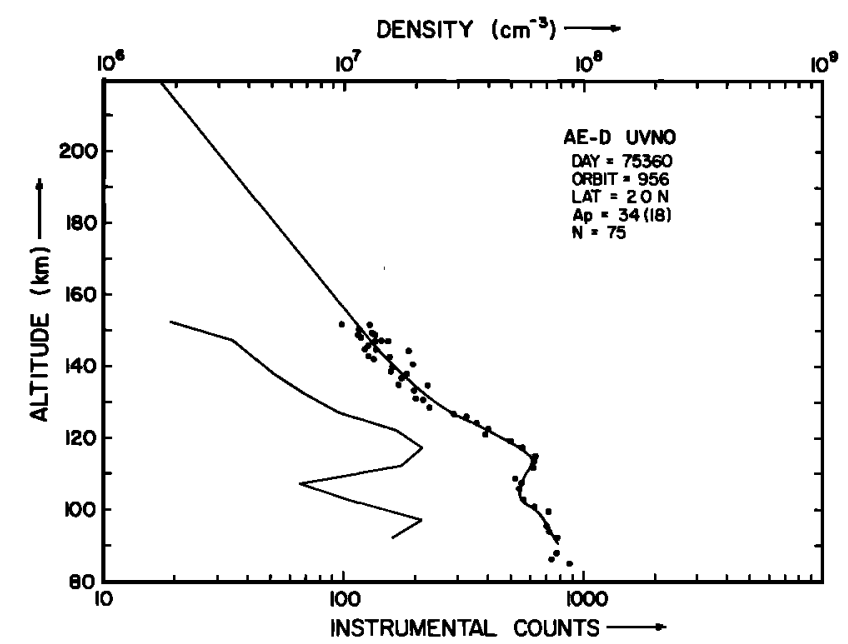

Fig. 6. See Figure 1 caption.

\subsection{Average NO Column Density Profiles (Contour Plot)}

In this section we will deal with a data set, as discussed earlier, in which the raw instrumental counts were corrected for Rayleigh scattering at lower altitudes, converted into slant column densities, and then sorted and averaged in $1^{\circ} \times 1 \mathrm{~km}$ latitude/altitude bins. The intrinsic latitude resolution of the data due to the length of the optical path and to the satellite motion is a few degrees. The results are shown in Figure 10. The contour plot shows several trends in the NO column density. The peak NO column densities are located at slightly over $100 \mathrm{~km}$ at all latitudes. The peak column densities increase from a minimum value of $10^{15} \mathrm{~cm}^{-2}$ near $20^{\circ} \mathrm{N}$ latitude (winter hemisphere) to $3 \times 10^{15} \mathrm{~cm}^{-2}$ near $70^{\circ} \mathrm{S}$ and to 9 $\times 10^{15} \mathrm{~cm}^{-2}$ near the northern terminator close to $50^{\circ} \mathrm{N}$. It seems quite possible that the actual maximum column density is located poleward of the terminator in the dark polar night. The latitudinal gradient is less pronounced at high altitudes, and the location of the latitudinal minimum shifts toward high winter latitudes (in agreement with the 200-km despun AE-D data presented by Cravens [1981]). The scale height of the NO column density distribution varies with both altitude and latitude. For instance, the scale height is rather small just above the peak near $50^{\circ} \mathrm{N}$, but at lower latitudes it becomes rather

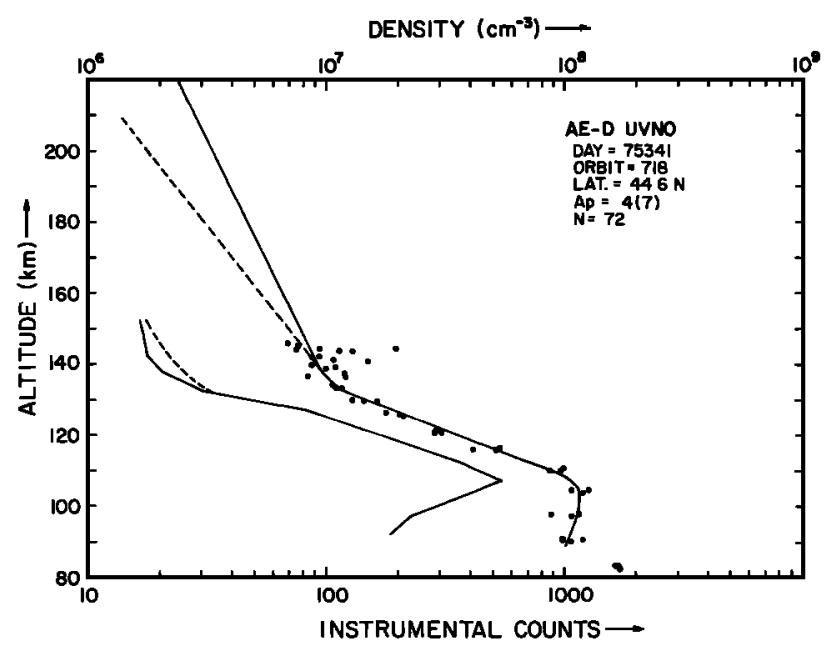

Fig. 8. See Figure 1 caption.

large near $140 \mathrm{~km}$, and the NO distribution appears to have a "ledge" near this altitude.

The measurements show a great deal of variability between successive individual profiles, and at a given latitude, between consecutive oribts. This variability is partially evident as irregularities in the contours in Figure 10. Small-scale variability in the data on the scale of a $1^{\circ}$ latitude bin is not primarily associated with latitude but is mostly variability in the observation times of the data put into that bin. The range of local times covered by the data set is small, but there were significant variations of magnetic activity level. Anomalously large NO values are observed near $50^{\circ} \mathrm{S}$ at all altitudes. The data for this latitude was primarily collected during midJanuary 1976, when AE-D perigee was in that vicinity. A magnetic storm occurred during this time period with the $A p$ index increasing from only 4 on January 9 up to values of 47 , 40 , and 13 on January 10,11 , and 12 , respectively. The $A p$ subsequently decreased to relatively quiet values. A close examination of the data shows that a moderate NO enhancement continued for a few days after the $A p$ returned to "normal." Figures 3 and 2 show some individual profiles before and during this magnetic storm, respectively. These individual NO profiles demonstrate that the NO densities in the lower thermosphere increase by a factor of 10 as a result of the magnetic storm.

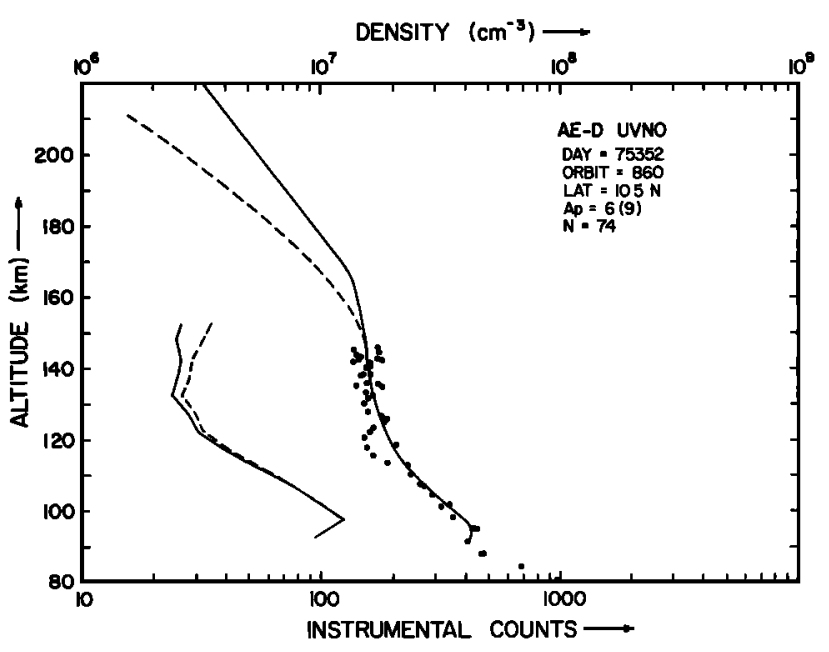

Fig. 7. See Figure 1 caption.

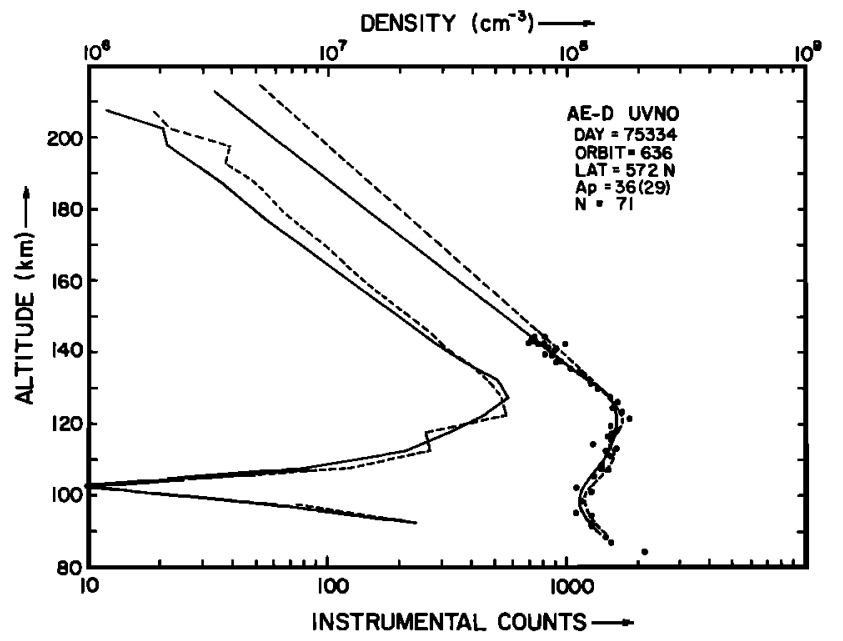

Fig. 9. See Figure 1 caption. 


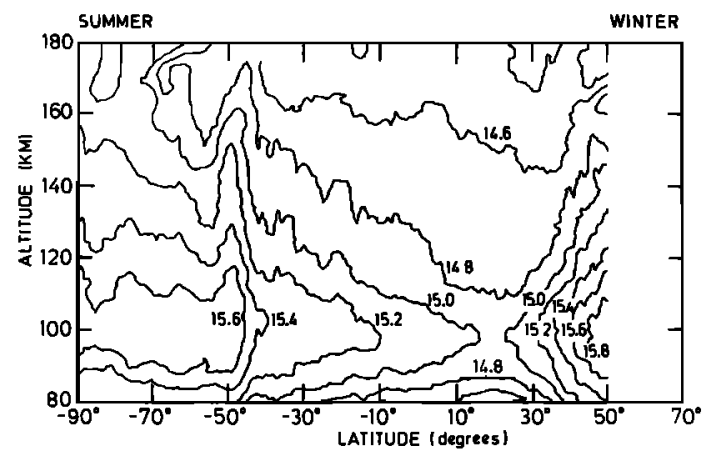

Fig. 10. Slant (horizontal) column density of NO as a function of altitude and latitude. Contours are labeled with the base $10 \log$ of the column density in units per square centimeter.

\subsection{Simplified Inversion}

Each of the individual profiles that went into Figure 10 was fitted with a simple two- or three-parameter function and then inverted, as described earlier. Figure 11 shows the latitudinal distribution of the maximum NO concentration deduced from these fits to the observations. The overall trends are the same as those which were evident at lower altitudes in Figure 10. The smallest values of the peak density, $n_{m}$, are located near $20^{\circ} \mathrm{N}$, and there is a gradual gradient in $n_{m}$ in the southern hemisphere and a sharper gradient in the northern hemisphere. Data from the mid-January storm were removed from this particular set of data, and so the localized enhancement near $50^{\circ} \mathrm{S}$ is not present. Even so, the amount of scatter is fairly large, especially in the northern mid-latitude region where the peak NO density changes from $10^{7}$ to $10^{8} \mathrm{~cm}^{-3}$.

Figure 12 displays the latitudinal distribution of $z_{m}$, the altitude of the peak NO concentration, obtained with the three-parameter fit. The $z_{m}$ values are scattered between 100 $\mathrm{km}$ and $120 \mathrm{~km}$, with a greater degree of scatter apparent in the northern latitudes. The latitudinal average of $z_{m}$ is 108.9 km.

\subsection{Average NO Number Density Profiles (Contour Plot)}

The instrumental counts were sorted and averaged using a $5^{\circ} \times 5 \mathrm{~km}$ latitude/altitude grid, as described earlier. Figure 13

SUMMER

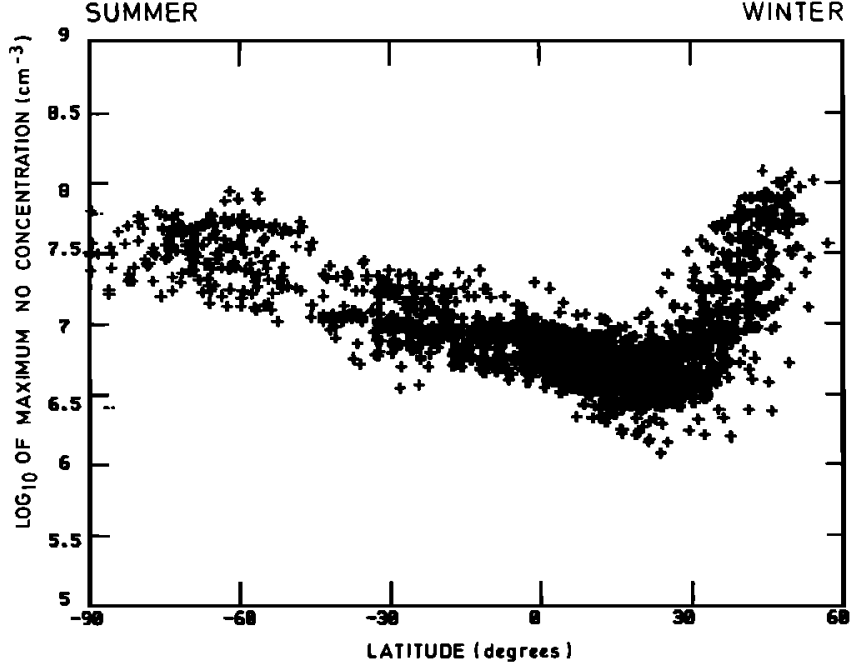

Fig. 11. Base $10 \mathrm{log}$ of the maximum NO concentration versus latitude. These densities were derived using a two- or three-parameter fit to each individual column density profile.
SUMMER

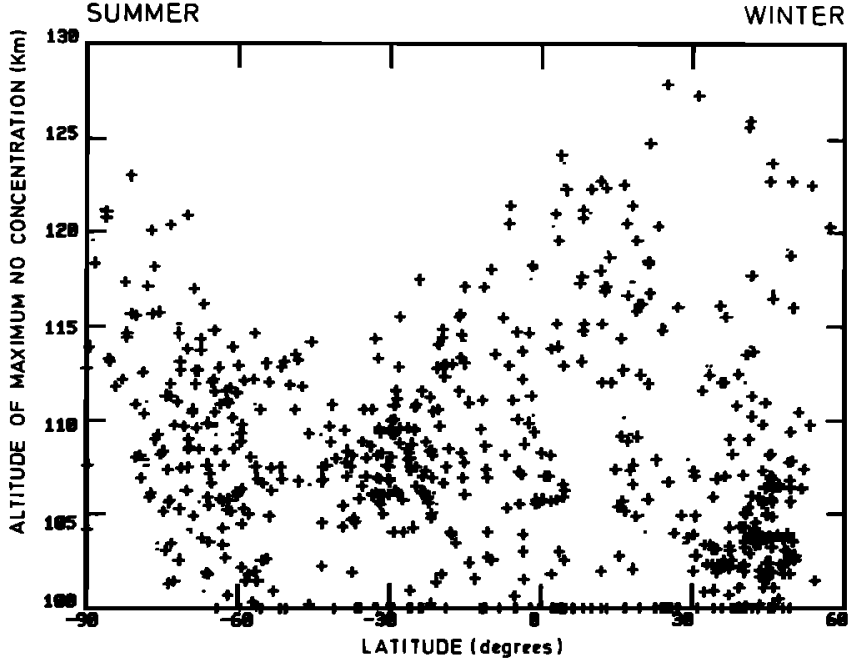

Fig. 12. Altitude of the maximum NO concentration versus latitude. These altitudes were derived using a three-parameter fit to each individual column density profile. Derived altitudes falling below 100 $\mathrm{km}$ were plotted at $100 \mathrm{~km}$.

displays the average counts and the standard deviation about this average as functions of altitude for the equatorial latitude bin. The number of data points in each altitude bin is also shown in this figure. There are 400 counts at $105 \mathrm{~km}$, and this is equivalent to $220 \mathrm{AE}-\mathrm{C}$ counts. AE-C usually observed $400-$ 500 counts at the equator; however, less NO (i.e., 200-300 counts) was observed during the morning local times which are relevant for comparing to AE-D data [Stewart and Cravens, 1978].

The $C$ profile shown in Figure 13, and similar profiles for the other latitudinal bins, were inverted to give NO number densities as a function of altitude (at $5-\mathbf{k m}$ intervals) using the procedures described earlier. There is little error introduced into the derived number density profile below $200 \mathrm{~km}$ by the required extrapolation for altitudes greater than $210 \mathrm{~km}$. In the same manner, average $C$ profiles and their respective number density profiles were obtained every $10^{\circ}$ in latitude. A three-point running average over altitude was performed at
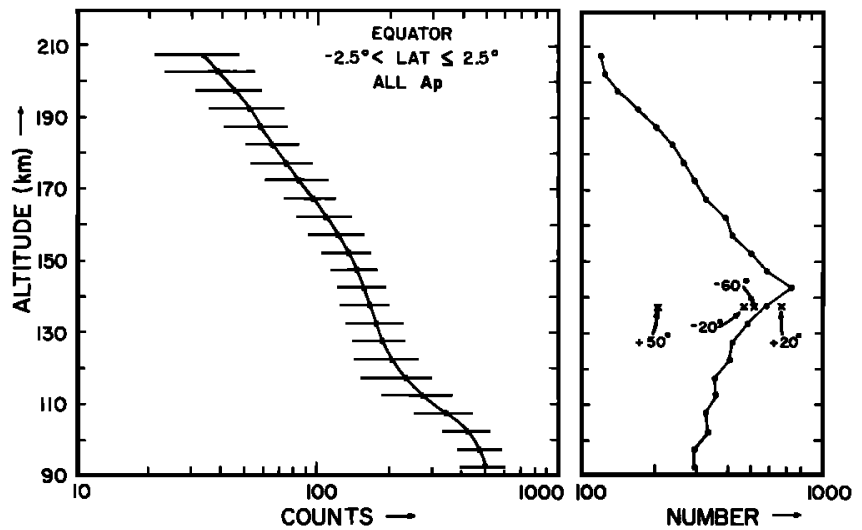

Fig. 13. The average instrumental counts as a function of altitude is shown for the equatorial latitude bin. The standard deviation in each 5-km altitude bin is also plotted. (Right) The number of data points in each altitude bin corresponding to the lefthand panel is indicated by the dots and solid line. The crosses are examples of the number of data points in the $137.5-\mathrm{km}$ altitude bin for several other latitude bins. 
each latitude in order to smooth the profiles, and the resulting data set was displayed as a latitude/altitude contour plot (Figure 14). The data incorporated into this contour plot represent a wide range of magnetic activity with an average $A p$ of 12.5. The magnetic storm of mid-January 1976 was not included in this data set.

The major morphological features seen in this contourplot are, not surprisingly, very similar to those seen in the higherresolution contour plot of slant column density (Figure 10), with the exception of the January magnetic storm, which was excluded from Figure 14. Obviously, then, the different procedures applied to the two different contour plots did not affect the major features of the data. However, both data sets are zonally and temporally averages of very large numbers of individual profiles, which restricts the possible interpretation of these data.

Consider Figure 14 in more detail. The peak NO density is located between 100 and $110 \mathrm{~km}$, with the lowest $z_{m}$ near $20^{\circ} \mathrm{N}$ latitude. The maximum NO concentration, $n_{m}$, has a latitudinal minimum near $20^{\circ} \mathrm{N}$ and has a sharp gradient toward the north and a more gradual gradient towards the south, in agreement with the results of the simplified inversion presented in Figure 11. In fact, the $n_{m}$ derived from the simple inversion procedure agrees quite well with the $n_{m}$ in Figure 14 at most latitudes. However, the simple inversion does give $n_{m}$ values that are about $50 \%$ less than the more sophisticated inversion for latitudes between about $20^{\circ} \mathrm{S}$ and $30^{\circ} \mathrm{N}$. An examination of either Figure 11 or 14, or some appropriate individual profiles, shows that in this range of latitudes the NO profile cannot accurately be described using a single scale height. Applying the simple two- or three-parameter fit and inversion to these more complicated profiles results in estimations of $n_{m}$ that are somewhat too small. For example, near the equator the NO distribution just above the peak has a scale height of approximately $20 \mathrm{~km}$, but between $120 \mathrm{~km}$ and $170 \mathrm{~km}$ or so the scale height is about $70 \mathrm{~km}$, and for altitudes above $170 \mathrm{~km}$ the profile is best described with a $30-\mathrm{km}$ scale height. There are some latitudes at which the average NO distribution can be described using a single scale height-the topside scale height is a reasonably constant $30 \mathrm{~km}$ or so at middle and high southerly latitudes. However, even at these latitudes, some of the individual profiles (if not the average) appear to have a more complicated structure (e.g., Figure 2) which the averaging procedure obscures.

The distribution of NO versus latitude for altitudes greater than $180 \mathrm{~km}$ or so has relatively simple structure-NO is more abundant in the summer hemisphere (S) than in the winter hemisphere $(\mathrm{N})$. This is in agreement with the despun AE-D data at $200 \mathrm{~km}$ reported by Cravens [1981].

We will now discuss a possible source of error in the derived NO densities at higher altitudes (above $170 \mathrm{~km}$ ). Victor and Dalgarno [1982] have suggested that dissociative photoionization of $\mathrm{N}_{2}$ is an important source of $\mathrm{N}^{+}\left({ }^{5} S_{2}\right)$ ions which radiate at 2139.7 and $2143.6 \AA$. They also suggested that highaltitude measurements of NO [i.e., Cravens, 1981] might be contaminated with the $2143-\AA$ emission which falls within the UVNO bandwidth for the 1-0 gamma band. The results of their calculations for the intensity of these $\mathrm{N}^{+}$features after being convolved with the UVNO sensitivity function give an equivalent $\mathrm{NO}$ density of $10^{6} \mathrm{~cm}^{-3}$ at $200 \mathrm{~km}$, which can be used to estimate the severity of the contamination of the NO measurements. This equivalent density would indeed indicate severe contamination, and in fact, some of the observed NO densities at $200 \mathrm{~km}$ (Figure 14) are as low as $3 \times 10^{5}$. Barth

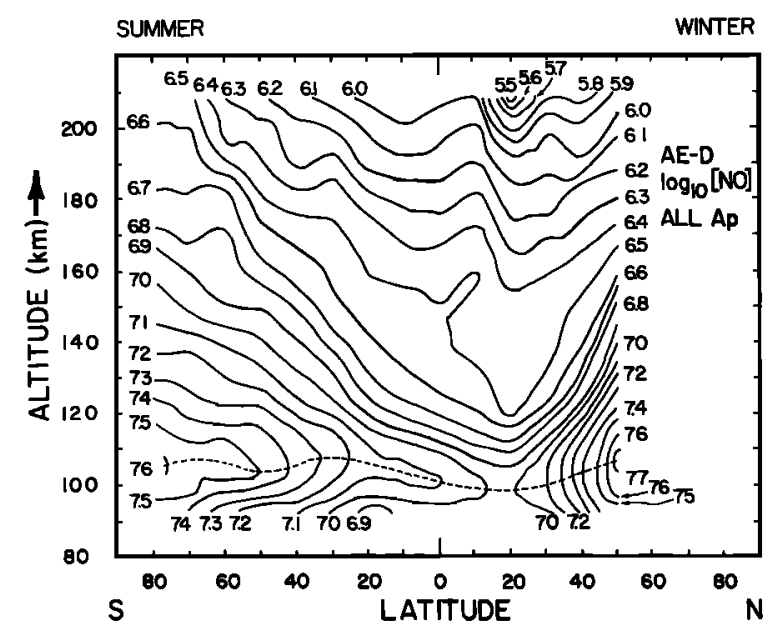

Fig. 14. Contour plot of the base $10 \log$ of the nitric oxide number density (per cubic centimeter) as a function of altitude and latitude. These values were calculated from zonally averaged and time-averaged intensity profiles such as that in Figure 13 using an inversion procedure described in the text. The dashed line indicates the location of the maximum NO concentration as a function of latitude.

and Steele [1982] measured on a rocket flight the $\mathrm{N}^{+}$dayglow at 2139 and $2143 \AA$ and found volume emission rates which were a factor of 6 smaller than those calculated by Victor and Dalgarno [1982]. This implies an equivalent NO density (as measured by the UVNO instrument including the effects of its sensitivity function) for the $\mathrm{N}^{+}$emissions of about $1.5 \times 10^{5}$ $\mathrm{cm}^{-3}$ at $200 \mathrm{~km}$ for solar minimum. This equivalent NO density was approximately adjusted for the differences in solar activity and solar zenith angle for the Barth and Steele [1982] measurements and the AE-D observations (lower solar activity). This degree of contamination of the UVNO measurements is not a significant problem at most latitudes, although where the derived NO densities are very small, as they are near $20-30^{\circ} \mathrm{N}$ in this paper at $200 \mathrm{~km}$, then these derived densities could be overestimated by about $50 \%$ and might actually be even smaller than the currently derived values.

\section{Discussion}

We have presented thermospheric NO densities measured by the UVNO instrument on the AE-D satellite for conditions of solar minimum, solstice, and approximately 0900 local time. The latitude and altitude distribution of nitric oxide was emphasized in this paper. We have reconfirmed the variations of NO with latitude and level of magnetic activity that the AE-C observed at $105 \mathrm{~km}$, as well as the trends observed at higher altitudes by AE-D during despun orbits. The present study has contributed to our understanding of the shape of the NO altitude profile and how it varies with latitude.

The contour maps (Figures 11 and 14) illustrate that even for average magnetic activity conditions there is a substantial latitudinal gradient of NO in the lower thermosphere, especially in the winter hemisphere. The individual profiles (Figures 1-9), as well as other studies such as those by Cravens and Stewart [1978] demonstrate that the amount of NO at middle and high latitudes strongly depends on the level of magnetic activity.

Zonally averaged and time-averaged pictures of the NO distribution such as those presented in this paper do not present a complete picture. An examination of even a timeaveraged global (i.e., both latitude and longitude) map of NO 


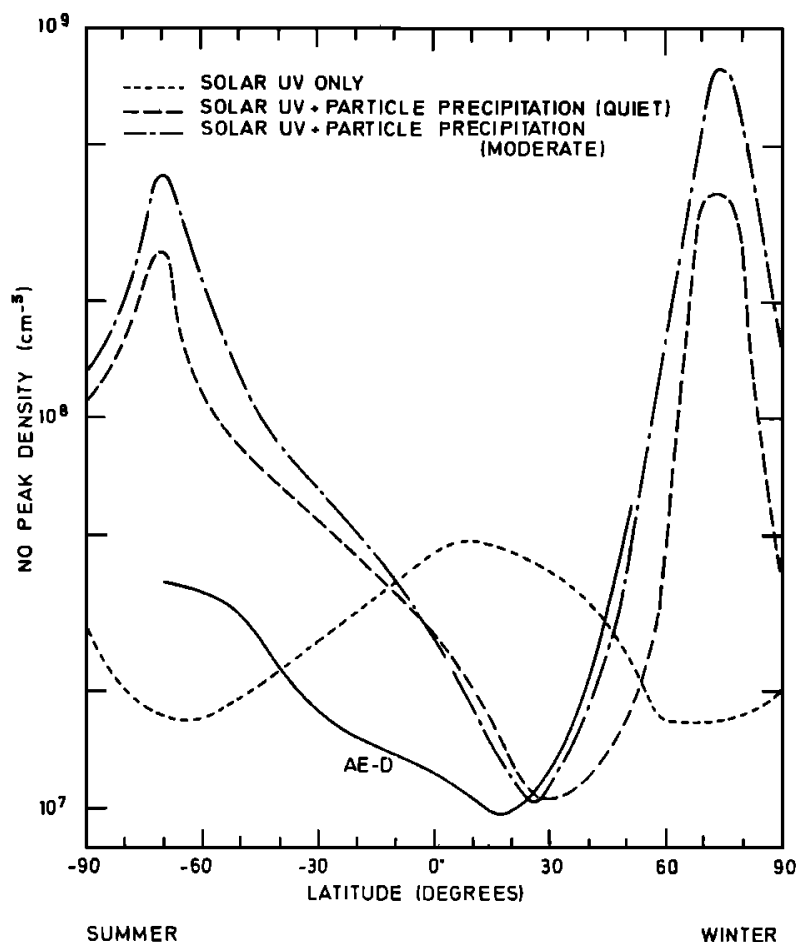

Fig. 15. Adapted from Figure 8 of Gérard et al. [1984]. Peak concentrations of nitric oxide are shown as a function of latitude. Theoretical results from the two-dimensional model fo Gérard et al are shown for quiet and moderate levels of magnetic activity, as well as for a case with only solar UV production. The heavy solid line represents peak concentrations taken from the AE-D contour plot (Figure 14).

at $105 \mathrm{~km}$ [Cravens and Stewart, 1978] indicates that the actual latitudinal gradient of NO at a fixed longitude is usually much sharper than the gradient of the zonally averaged NO. Time-averaging NO over periods of time covering a range of magnetic activity also softens the latitudinal gradient, whereas the gradient for a specific time would be sharper.

Nitric oxide has been measured on rocket flights using either the resonance scattering technique during daylight conditions or the ion composition analysis technique. The peak NO concentrations measured by rocket experiments for low and middle-latitudes are in general agreement with those presented in this paper for comparable solar activity, magnetic activity, and local time conditions. The shapes of the low- and mid-latitude NO profiles measured by rockets and AE-D are similar. For instance, NO was measured at $38.7^{\circ} \mathrm{N}$ latitude in December and from 110 to $300 \mathrm{~km}$ during very quiet magnetic conditions on the rocket flight of Thomas [1978]. The peak density of $6.2 \times 10^{6} \mathrm{~cm}^{-3}$ is comparable to the low values reported in this paper for comparable latitudes and quiet magnetic conditions. The shape of this rocket profile above the peak exhibits a pronounced plateau above $120 \mathrm{~km}$ and is very similar to the shapes of the altitude profiles reported here for low latitudes and/or for relatively quiet conditions (see Figure 5,10 , or 14 ).

The rocket data for high summer latitudes show peak NO densities which are typically between $10^{8}$ and $10^{9} \mathrm{~cm}^{-3}$ [c.f. Gérard et al., 1983]. These values are significantly greater than those reported in this paper for these latitudes. In the dark polar night, NO concentrations in excess of $10^{9} \mathrm{~cm}^{-3}$ have often been observed by rocket experiments; however, the UVNO experiments on AE-C and AE-D, or any other experi- ment using the resonance scattering technique, were incapable of measuring NO in this region.

There are two plausible explanations for the discrepancy between the rocket and AE-D NO densities in the summer polar region. First, most of these rocket flights were flown directly into bright auroral arcs, where theoretically the NO concentration is expected to be enhanced [Gérard and Barth, 1977], and thus these experiments are biased toward higher than average NO densities. Second, satellite experiments, such as the one reported in this paper, have a horizontal spatial resolution of a few degrees and therefore only measure the average NO concentration. Rocket measurements of NO are essentially either from in situ ion composition measurements or from upward looking resonance scattering observations. In both cases the geometry is such that the horizontal resolution is much smaller than satellite measurements. Horizontal resolution is not a problem at low and middle latitudes, where the effective spatial scale of the NO variations is greater than a few degrees, even for rather large latitudinal gradients; however, the relevant horizontal spatial scale in the auroral region is significantly smaller than a few degrees. Hence the NO observed by the satellite is averaged over a few degrees, and these densities should be less than those measured in aurorally active regions by rockets.

One-dimensional theories of thermospheric odd nitrogen [e.g., Oran et al., 1975; Rusch et al., 1977; Cravens et al., 1979] can explain neither the shape nor the magnitude of the measured altitude and latitude distribution of NO, except at low latitudes and/or during quiet times. The two-dimensional model of Gerard et al. [1984], which includes horizontal and vertical transport of odd nitrogen, is capable of at least qualitatively reproducing the most important features of the observed NO distribution.

Figure 8 in the Gerard et al. paper (this figure is partially reproduced in Figure 15 of this paper) is a comparison of three model calculations of the latitudinal distribution of the peak NO density (for three levels of magnetic activity) with the peak density calculated from the observed zonally averaged densities from Figure 14 in this paper. Except at high summer (S) latitudes, there is better than a factor of 2 quantitative agreement between the observed and the model peak NO densities. In particular, both the theoretical and experimental densities have minimum near $20^{\circ}$ or $30^{\circ} \mathrm{N}$ (winter) latitude, and both exhibit a steeper latitudinal gradient in the winter than in the summer hemisphere.

The shape of the theoretical altitude distribution of NO is also in fairly good agreement with the observations. Both the theory and observations indicate that $\mathrm{NO}$ at $200 \mathrm{~km}$ is more abundant in the summer hemisphere than in the winter. At intermediate altitudes the situation is somewhat more complicated, but the theory does give some indication of the $120-\mathrm{km}$ to $180-\mathrm{km}$ low-latitude plateau in the NO concentration that appears in the data and which has been discussed earlier in this paper. There is also qualitative agreement between theory and experiment concerning the shapes of the vertical profiles in the vicinity of the maximum NO concentration. Figures 10 and 14 both show that the peak in the summer hemisphere is rather narrow, or sharp, and is well defined with a rapid decrease toward the mesospheric "dip" at lower altitudes. And the peak in the winter hemisphere is rather broad with only a gradual drop-off in density below the peak. The twodimensional model of Gérard et al. correctly reproduces these observational features qualitatively. The NO profiles measured by rocket experiments also confirm this behavior of the 
NO near and below the maximum. Solomon et al. [1982] have explained this behavior by a combination of lower predissociation rates in the winter and larger eddy diffusion coefficients in the winter than in the summer.

In summary, the UVNO experiment measured the altitude and latitude distribution of nitric oxide in the thermosphere. These measurements are characterized by morning local time, solar cycle minimum, and relatively quiet magnetic conditions. Both individual NO profiles and zonally and time-averaged latitude/altitude distributions were presented. Future experimental studies should combine the latitude (or magnetic latitude) and longitude coverage of the 105-km AE-C studies [Cravens and Stewart, 1978; Stewart and Cravens, 1978] with the altitude coverage of this AE-D study. That is, threedimensional data sets should be constructed and analyzed. More sophisticated and higher-resolution inversion techniques should be applied in the auroral regions in order to resolve some of the differences between satellite and rocket measurements of high-latitude NO. The AE-C and D data sets contain more spatial and temporal information than we have utilized in this paper, and it would be instructive to apply to these data the more sophisticated techniques just discussed. Of course, three-dimensional theoretical models will then be required to interpret these more complex data sets.

Acknowledgments. This work was supported by NASA grant NGR 23-005-015. One of us (J.C.G.) is supported by the Belgian Foundation for Scientific Research and (FRFC) grant 2-4507-82. This research was supported in part by NATO Cooperative research grant 166-84. The authors thank D. Roble and S. Q. Rudy for their help with the data analysis.

The Editor thanks R. P. McCoy and G. A. Victor for their assistance in evaluating this paper.

\section{REFERENCES}

Barth, C. A., and R. E. Steele, Rocket observations of the NII 2143A Dayglow, Geophys. Res. Lett., 9, 559-561, 1982.

Barth, C. A., D. W. Rusch, and A. I. Stewart, The UV nitric-oxide experiment for Atmosphere Explorer, Radio Sci., 8, 379, 1973.

Cravens, T. E., Nitric oxide gamma band emission rate factor, Planet. Space Sci., 25, 369, 1977.

Cravens, T. E., The global distribution of nitric oxide at $200 \mathrm{~km}, J$. Geophys. Res., 86, 5710-5714, 1981.

Cravens, T. E., and A. I. Stewart, Global morphology of nitric oxide in the lower $E$ region, J. Geophys. Res., 83, 2446, 1978.

Cravens, T. E., J.-C. Gérard, A. I. Stewart, and D. W. Rusch, The latitudinal gradient of nitric oxide in the lower thermosphere, $J$. Geophys. Res., 84, 2675, 1979.
Gerrard, J.-C., and C. A. Barth, High-latitude nitric oxide in the lower thermosphere, J. Geophys. Res., 82, 674, 1977.

Gérard, J.-C., E. Deneye, and V. Singh, Rocket measurements and modeling of the nitric oxide distribution in the thermosphere and lower mesosphere, Sixth ESA Symposium on European Rocket and Balloon Programmes, Spec. Publ. 183, pp. 123-130, Eur. Space Agency, Neuilly, France, 1983.

Gérard, J.-C., R. G. Roble, D. W. Rusch, and A. I. Stewart, The global distribution of thermospheric odd nitrogen for solstice conditions during solar cycle minimum, J. Geophys. Res., 89, 1725-1738, 1984.

McCoy, R. P., Thermospheric odd nitrogen, 1 , NO, $N\left({ }^{4} S\right)$, and $O\left({ }^{3} P\right)$ densities from rocket measurements of the NO $\delta$ and $\gamma$ bands and the $\mathrm{O}_{2}$ Herzberg I bands, J. Geophys. Res., 88, 3197-3205, $1983 a$.

McCoy, R. P., Thermospheric odd nitrogen, 2, Comparison of rocket observations with a diffusive transport chemical model, J. Geophys. Res., 88, 3206-3210, $1983 b$.

Oran, E. S., P. S. Julienne, and D. F. Strobel, The aeronomy of odd nitrogen in the thermosphere, J. Geophys. Res., 80, 3068, 1975.

Roble, R. G., and J. M. Gary, The effect of horizontal transport on auroral NO densities, Geophys. Res. Lett., 6, 703, 1979.

Rusch, D. W., Satellite ultraviolet measurements of nitric oxide fluorescence with a diffusive transport model, J. Geophys. Res., 78, 5676, 1973.

Rusch, D. W., and C. A. Barth, Satellite measurements of nitric oxide in the polar region, J. Geophys. Res., 80, 3719, 1975.

Rusch, D. W., and W. E. Sharp, Nitric oxide delta band emission in the earth's atmosphere: Comparison of a measurement and a theory, J. Geophys. Res., 86, 1011, 1981.

Rusch, D. W., T. E. Cravens, G. R. Carignan, A. I. Stewart, and J.-C Gerard, A theoretical model of odd nitrogen in the earth's thermosphere and mesosphere (abstract), Eos. Trans. AGU, 58, 1198, 1977.

Solomon, S., P. J. Crutzen, and R. G. Roble, Photochemical coupling between the thermosphere and the lower atmosphere, I, Odd nitrogen from 50 to $120 \mathrm{~km}, J$. Geophys. Res., 87, 7206, 1982.

Stewart, A. I., and T. E. Cravens, Diurnal and seasonal effects in $E$ region low-latitude nitric oxide, J. Geophys. Res., 83, 2453, 1978.

Thomas, R. J., A high-altitude rocket measurement of nitric oxide, $J$. Geophys. Res., 83, 513, 1978.

Victor, G. A., and A. Dalgarno, The 2145A dayglow and the high altitude nitric oxide density, Geophys. Res. Lett., 9, 866-868, 1982.

T. E. Cravens, Space Physics Research Laboratory, Atmospheric and Oceanic Science Department, The University of Michigan, 2455 Hayward, Ann Arbor, MI 48109.

J.-C. Gérard, Institut d'Astrophysique, Université de Liège, Cointe-Liège, 4200 Belgium.

M. LeCompte, D. W. Rusch, and A. I. Stewart, Laboratory for Atmospheric and Space Physics, Department of Astrophysical, Planetary, and Atmospheric Sciences, University of Colorado, Boulder, CO 80309.

(Received November 15, 1984; revised May 31, 1985; accepted June 28,1985 .) 